\title{
A reference current source with a low temperature coefficient
}

\author{
DongYang Han ${ }^{1, a}$,XiaoNing Xin ${ }^{2}$ and GuoFeng Liu ${ }^{3}$ \\ ${ }^{1}$ College of Information Science and Engineering, Shenyang University of Technology, China \\ ${ }^{2}$ College of Information Science and Engineering, Shenyang University of Technology, China \\ ${ }^{3}$ College of Information Science and Engineering, Shenyang University of Technology, China
}

\begin{abstract}
In view of the high demand for low temperature coefficient reference current sources in integrated circuits, the advantages and disadvantages of several typical reference current sources in circuit structure and temperature characteristics are compared. In this paper, a low temperature coefficient reference current source is designed. Based on Hua Hong HHNECGE 0.35um process, the output current is $0.4 \mathrm{uA}$, the temperature range is $-40^{\circ} \mathrm{C} \sim 125^{\circ} \mathrm{C}$, and the temperature coefficient is $7.6 \mathrm{ppm} /{ }^{\circ} \mathrm{C}$ under typical process angle. Other processes The temperature coefficient under the corner is at $10 \mathrm{ppm} /{ }^{\circ} \mathrm{C}$.
\end{abstract}

\section{Introduction}

The reference voltage source and the reference current source form a reference circuit. The roles of the two structures in the circuit are to provide a voltage reference and a current reference. As the integrated circuit develops, the reference current source becomes an important module in the integrated circuit. As an essential part of analog integrated circuits. High-performance analog circuits must be supported by high-quality, high-stability voltage and current bias circuits. Its performance directly affects the power consumption and power supply rejection ratio of the circuit, open loop gain and temperature characteristics. Nowadays, various current reference source implementations emerge in an endless stream. The most common current sources are proportional reference current source, $\mathrm{V}_{\mathrm{BE}}$ type reference current source, PTAT reference current source, and zero temperature coefficient reference current source. Their characteristics are different. The main principle of the reference current source is to use the positive temperature coefficient current and the negative temperature coefficient current to obtain the zero temperature coefficient current, which is also the basic principle of the band gap[1]. Generally, the positive temperature coefficient current is taken from the PTAT (proportional to absolute temperature) current in the bandgap reference circuit, and the negative temperature coefficient current is generated by different principles. This paper discusses the design method of a new low temperature coefficient reference current source circuit.

\section{Reference current source structure}

The reference current source is the core of the analog circuit and the most commonly used module[2]. Its main function is to generate a current independent of the temperature coefficient and then mirror it to other circuits through the current mirror. Figure2.1 shows the most basic reference current generating circuit. It consists of two NMOS transistors M1,M2 and a resistor R1. The current mirror is configured to generate a mirror circuit to output current to the outside. The resistance value flowing through $\mathrm{M} 1$ is

$$
\mathrm{I}=\frac{\mathrm{V}_{\mathrm{DD}}-\mathrm{V}_{\mathrm{THN}}}{\mathrm{R}_{0}}
$$

If we take the derivative of equation (1) with respect to temperature, we get equation (2)

$$
\frac{\partial \mathrm{I}}{\partial \mathrm{T}}=\frac{\mathrm{R}_{0} \times \frac{\partial \mathrm{V}_{\mathrm{THN}}}{\partial \mathrm{T}}+\left(\mathrm{V}_{\mathrm{DD}}-\mathrm{V}_{\mathrm{THN}}\right) \times \frac{\partial \mathrm{R}_{0}}{\partial \mathrm{T}}}{\mathrm{R}_{0}^{2}}
$$

Since $R_{1}$ and $\partial V_{T H N}$ in equation (2) all change with temperature, the temperature characteristics of the circuit will be poor. The disadvantage of this circuit is that the magnitude of the current is affected by the power supply voltage, the power supply rejection is poor, and the temperature characteristics of the current are poor.

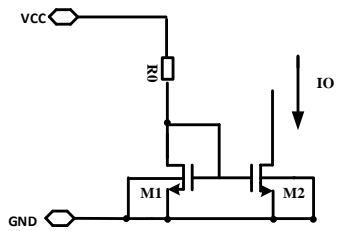

Figure 2.1 Basic current source

As shown in Figure 2.2, there are two paths from the power supply $V_{D D}$ to point $A$ in the figure. One is the $B E$ junction through $R_{1}, Q_{2}$, the $B E$ junction of $Q_{3}$ and $R_{4}$ to $\mathrm{A}$, the other is the $\mathrm{BE}$ junction through $\mathrm{Q}_{1}$, the $\mathrm{BE}$

\footnotetext{
* Corresponding author: a Dong Yang Han: 862740050@qq.com
} 
junction of $Q_{4}$ and $R_{5}$ to $A$, if all base currents are ignored, then there is

$$
\begin{aligned}
& V_{A}=V_{D D}-I_{R 1} R_{1}-V_{B E 2}-V_{B E 3} \\
& V_{A}=V_{D D}-V_{B E 1}-V_{B E 4} \\
& I_{R 1} R_{1}=\left(V_{B E 1}-V_{B E 3}\right)+\left(V_{B E 4}-V_{B E 2}\right)
\end{aligned}
$$

If $\beta$ is large enough, the base current can be neglected.

$$
\mathrm{I}_{1}=\mathrm{I}_{3}, \mathrm{I}_{2}=\mathrm{I}_{4}
$$

The lateral PNP tube in the process has a high $\beta$ value, which just satisfies this condition, so

$$
\mathrm{I}_{4}=\mathrm{I}_{\mathrm{R} 1}=\frac{2 \mathrm{~V}_{\mathrm{T}} \ln 4}{\mathrm{R}_{1}}
$$

An outstanding advantage of this circuit is that the currents of the two branches are not required to be equal, and the "inactive" current $I_{3}$ can be much smaller than the effective current $\mathrm{I}_{4}$ supplied to the load. The power supply rejection of this circuit depends on the resistor R2. The larger the R2, the better the voltage suppression capability. It can be seen that $\mathrm{I}_{4}$ is basically independent of the supply voltage but temperature dependent.

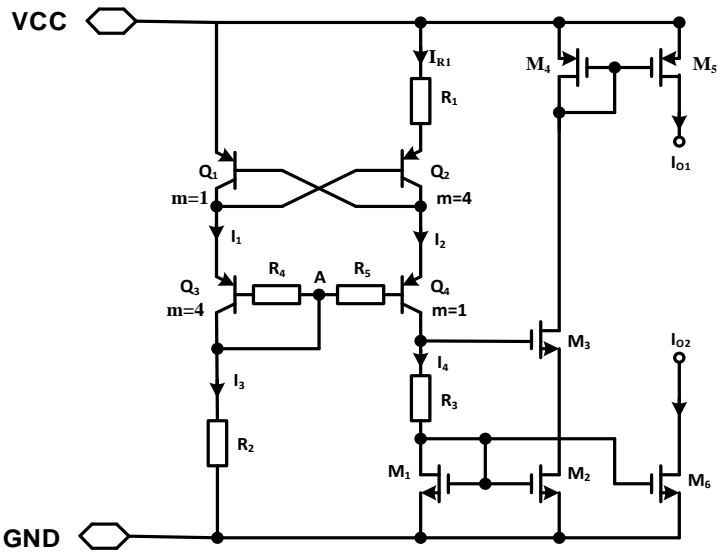

Figure 2.2 A current source based on PATA voltage

This current source uses the PTAT voltage to generate a reference current across the resistor, making it easier to generate a reference current source with a positive temperature coefficient[3]. The temperature coefficient of the current is related to the temperature coefficient of the resistor. If a negative temperature coefficient resistor is used in the PTAT current source, the temperature coefficient of the current will be large[4]. Using a positive temperature coefficient resistor reduces the effect of temperature on the current. However, there is no suitable positive temperature coefficient resistance in the BCD350GE process. The temperature coefficient of the nwell type resistor is too large, and the PTAT voltage with a high temperature coefficient is needed to cancel, and the process deviation of this resistor is $\pm 21 \%$, and the occupied area is also large. . The rnpoly type resistor block value is too small, the block resistance is only $9 \Omega$, and the process deviation is $\pm 33 \%$. It is also not suitable for this current source. The use of two temperature coefficient resistors in series may have a certain effect, but the actual effect is limited because the matching relationship cannot be guaranteed[5]. The reference current is generally not used directly, but is transformed by some kind of circuit, and it is a more common method to improve the temperature coefficient during the conversion process.
In addition to the PTAT type current source based on $\Delta V_{\mathrm{BE}}$, the $\mathrm{V}_{\mathrm{BE}}$ voltage can also be used to generate a current independent of the power supply voltage on the resistor, referred to as " $\mathrm{V}_{\mathrm{BE}}$ type current source".

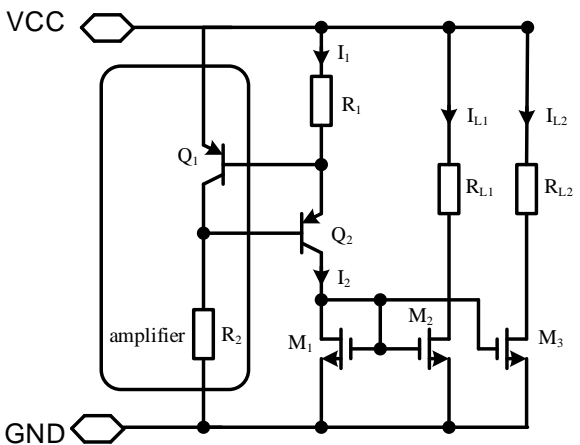

Figure 2.3 PNP tube $\mathrm{V}_{\mathrm{BE}}$ current source

Figure 2.3 is a circuit that uses a $V_{B E}$ voltage of a PNP tube to generate a reference current across a resistor. This circuit uses a negative feedback mechanism to improve the supply voltage suppression capability.

$Q_{1}$ and $R_{2}$ in Figure 2.3 form an amplifier. When $I_{1}$ increases, the base voltage of $Q_{1}$ drops, which is equivalent to the input voltage drop of the amplifier. Therefore, the collector voltage of $Q_{1}$ rises, because $Q_{1}$ is connected to $Q_{2}$. The base of the pole causes the $I_{1}$ to drop. Since the higher the $\mathrm{R}_{2}$, the larger the amplification factor of the amplifier, the better the constant current effect. In addition, increasing $R_{2}$ can reduce the ineffective current flowing from $R_{2}$, so $R_{2}>>R 1$ should be designed.

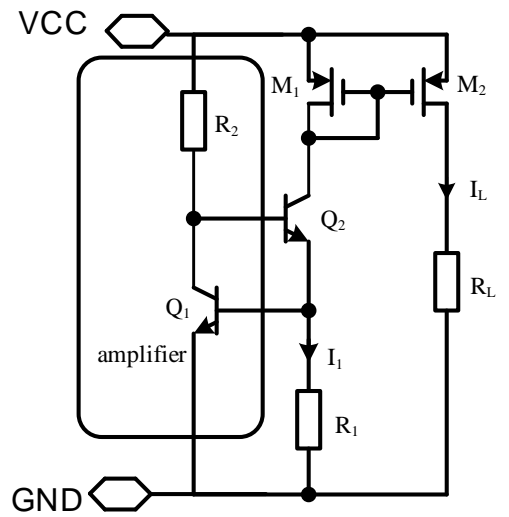

Figure 2.4 NPN tube $V_{B E}$ current source

Figure 2.4 shows a similar circuit implemented using an NPN tube. "In any circuit, there is always a complementary symmetrical circuit based on the same principle," that is, if you design a circuit with a certain function using PNP and NMOS, then replace all PNPs with NPN. By replacing all NMOS transistors with PMOS transistors and connecting them in a symmetrical relationship, the resulting circuit has a similar function to the original circuit. Compared with the PTAT voltage, the value of $\mathrm{V}_{\mathrm{BE}}$ is relatively large, and a large resistor is required, which is a disadvantage of such a current source. Since $V_{B E}$ has a negative temperature coefficient, a negative temperature coefficient resistor is needed to improve the temperature characteristics of the current. When implemented in the BCD350GE process, the two best-performing polycrystalline resistors rrpolym and rrpolyh are just negative temperature coefficients, of 
which the rrpolyh type resistor has a high block value and a large negative temperature coefficient, which is used in this circuit Suitable. Since $V_{B E}$ drops rapidly with temperature, even with a rrpolyh resistor with a large negative temperature coefficient, the current will exhibit a negative temperature coefficient.

\section{New current source structure}

The temperature characteristics of the current are determined by both the temperature characteristics of the voltage and the temperature characteristics of the resistor. Obviously, adjustment of any one may achieve temperature compensation. Discussion the temperature compensation problem cannot be started from the ideal situation. The process deviation problem must be considered, and the method that is not sensitive to the process deviation is a practical method. A current temperature compensation idea, The relationship between the collector current and $\mathrm{V}_{\mathrm{BE}(\mathrm{T})}$ is

$$
\mathrm{I}_{\mathrm{C}}(\mathrm{T})=\mathrm{I}_{\mathrm{S}}(\mathrm{T}) \exp \left[\frac{\mathrm{qV_{ \textrm {BE } } ( \mathrm { T } )}}{\mathrm{KT}}\right]
$$

$$
\mathrm{V}_{\mathrm{BE}(\mathrm{T})}=\frac{\mathrm{kT}}{\mathrm{q}} \ln \frac{\mathrm{I}_{\mathrm{c}}(\mathrm{T})}{\mathrm{I}_{\mathrm{S}}(\mathrm{T})}=\frac{\mathrm{kT}}{\mathrm{q}} \ln \mathrm{I}_{\mathrm{c}}(\mathrm{T})-\frac{\mathrm{kT}}{\mathrm{q}} \ln \mathrm{I}_{\mathrm{S}}(\mathrm{T})
$$

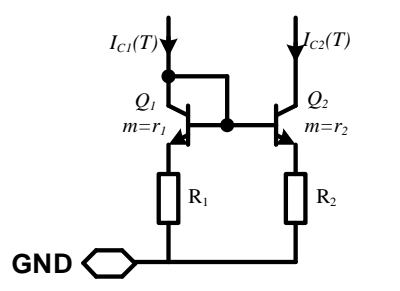

Figure 2.2 Circuit connection relationship According to the circuit connection relationship, there is $\mathrm{I}_{\mathrm{C}}(\mathrm{T}) \mathrm{R}_{1}(\mathrm{~T})-\mathrm{I}_{\mathrm{C} 2}(\mathrm{~T}) \mathrm{R}_{2}(\mathrm{~T})+\frac{\mathrm{kT}}{\mathrm{q}} \ln \frac{\mathrm{I}_{\mathrm{C} 1}(\mathrm{~T})}{\mathrm{r}_{1}}=\frac{\mathrm{kT}}{\mathrm{q}} \ln \frac{\mathrm{I}_{\mathrm{c} 2}(\mathrm{~T})}{\mathrm{r}_{2}}(9)$

As can be seen from (9), adjusting $\mathrm{I}_{\mathrm{c} 1}(\mathrm{~T}), \mathrm{R}(\mathrm{T}), \mathrm{r}_{1}$ and $r_{2}$ can change $I_{c 2}(T)$. According to this idea, the low temperature coefficient reference current source as shown in Figure 3.1 is designed.

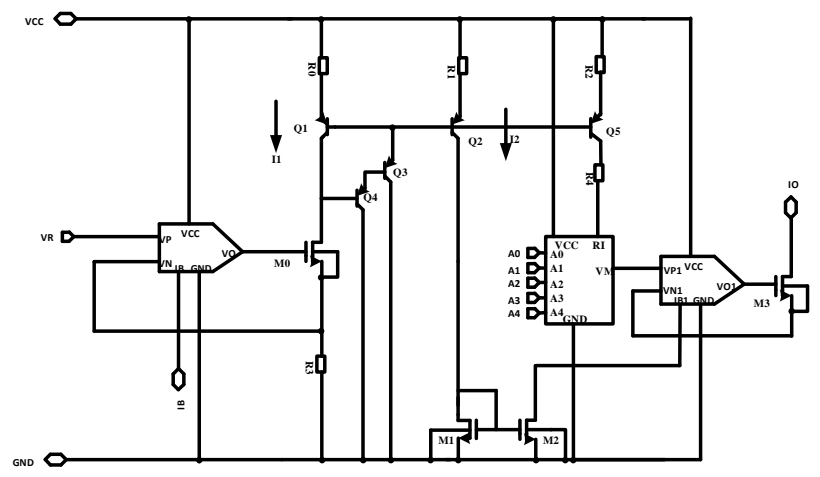

Figure 3.1New low temperature coefficient reference current source integrated circuit

\section{Circuit simulation result}

After the circuit design is completed, we completed the layout design according to the design rules required by the BCD350GE process. Figure 4.1 shows the simulation using Cadence and Hspice software. The simulation results show that the process angle is changed and the temperature coefficient of the current remains basically unchanged as shown in Figure 4.2, 4.3. Based on Hua Hong HHNECGE 0.35um process, the output current is $0.4 \mathrm{uA}$, the temperature range is $-40^{\circ} \mathrm{C} \sim 125^{\circ} \mathrm{C}$, and the temperature coefficient is $7.6 \mathrm{ppm} /{ }^{\circ} \mathrm{C}$ under typical process angle. Other processes The temperature coefficient under the corner is at $10 \mathrm{ppm} /{ }^{\circ} \mathrm{C}$.

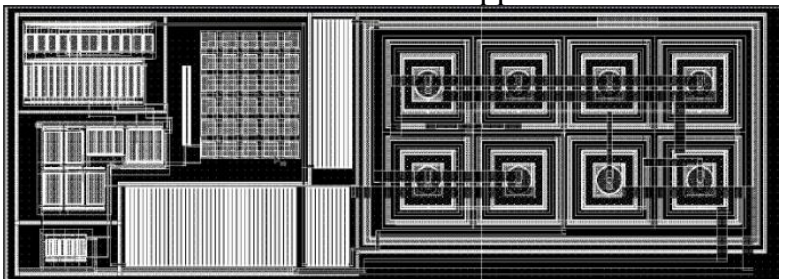

Figure 4.1. Low Temperature Coefficient Reference Current Source Layout

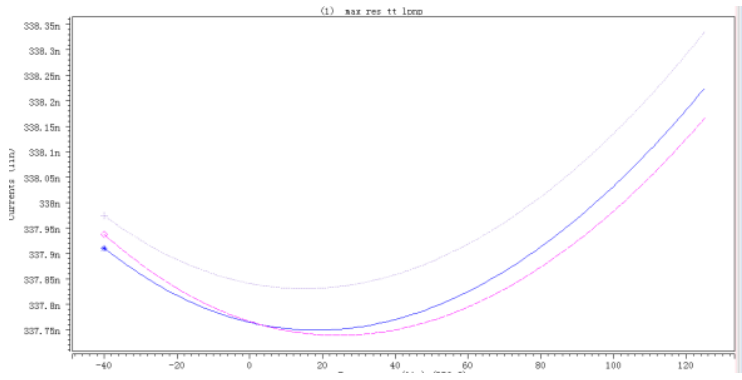

Figure 4.2. Temperature coefficient simulation waveform

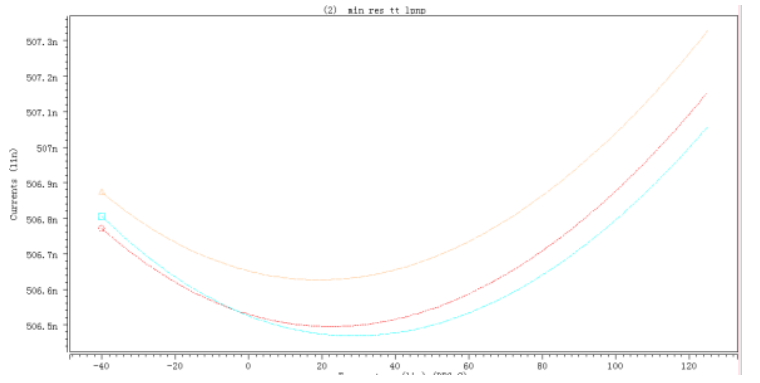

Figure 4.3. Temperature coefficient simulation waveform

\section{Conclusion}

In this paper, through the research and analysis of the reference current source, a low current coefficient reference current source is designed to ensure that the temperature coefficient remains basically unchanged at any process angle. After simulation, the reference current source meets the application requirements.

\section{References}

1. O.Cerid, S.Bakir, G. Dundar, Novel CMOS reference current genterator ,International Journal of Electronics , Vol.78, June 1995, pp.1113-1118. 
2. H.J.Oguey, D. Aebischer, CMOS current reference without resistance, IEEE Jounal of Solid-State Circuits,Vol.32,NO.7,July 1997,pp.1132-1135.

3. F.Fiori,P.S. Crovetti,A New Compact TemperatureCompensated CMOS Reference, IEEE Transactions on Circuits and Systems- II, Vol.52,No.11,November 2005,pp.724-728.

4. Lu Yang, B. Zhang and other ,A 1.8-V 0.7ppm/derg.C high order temperature-compensated CMOS current reference,Analog Intergrated Circuits,Vol.51,No.3,Jan.2007,p.175-179.

5. A. Djemouai, M. Sawan, M. Slamani, "New Circuit Techniques based on a High performance Frequencyto-Voltage Conver. ” ,Proc.ICECS'99, pp.1316,1999,Pafos,Cyprus. 